${ }^{1} \mathrm{H}$ Altunhan, ${ }^{2} \mathrm{~A}$ Annagür, ${ }^{3} \mathrm{M}$ Konak, ${ }^{4} \mathrm{~S}$ Kurban, ${ }^{4} \mathrm{j}$ Mehmetoğlu, ${ }^{3} \mathrm{R}$ Örs. ${ }^{1}$ Abant lzzet Baysal University, Medical Faculty, Department of Neonatology, Bolu; ${ }^{2}$ Selcuk University, Selcuklu Medical Faculty, Department of Neonatology; ${ }^{3}$ Konya University, Meram Medical Faculty, Department of Neonatology; "Konya University, Meram Medical Faculty, Department of Clinical Biochemistry, Konya, Turkey

Aim To investigate the oxidant-antioxidant status in babies born to preeclamptic mothers (BBPM).

Method The PON-1(Paraoxonase), TAS(Total Antioxidant Status) and TOS(Total Oxidant Status) levels were measured in the cord blood and venous blood (7day) of babies born to preeclamptic $(n=31)$ and normotensive $(\mathrm{N}=25)$ mothers.

Results There was no difference between the two groups in terms of PON-1 and TOS levels in the cord blood and venous blood. However, the cord blood TAS levels were higher in BBPM ( $p=0.001)$; the TAS levels in the venous blood were higher in the control group $(p=0.021)$. Furthermore, the cord blood PON-1 levels of babies born to severely preeclemptic mothers $(n=18)$ were higher than those of babies born to mildly-moderately preeclamptic mothers $(n=13)$ $(p=0.042)$. There was no difference between cord blood TAS and TOS of babies born to severely and mildly-moderately preeclamptic mothers and the venous blood PON-1, TAS and TOS levels.

Conclusion The increased TAS level that was found in the cord blood of BBPM compared to that of the control group indicates that fetus is protected against oxidative damage caused by increased oxidative stress of the mother. Furthermore, the fact that the cord blood PON-1 level of babies born to severely preeclamptic mothers was higher than that of babies born to mildly-moderately preeclamptic mothers indicates the presence of a positive correlation between the severity of oxidative stress in the mother and the antioxidant protection of the baby. Our study is the first in the literature investigating PON-1 in BBPM.

\section{ASSOCIATION OF DELIVERY TYPE WITH POSTPARTUM DEPRESSION AND MATERNAL ATTACHMENT}

doi:10.1136/archdischild-2012-302724.1289

${ }^{1} \mathrm{~S}$ Hergüner, ${ }^{2} \mathrm{E}$ Çiçek, ${ }^{3} \mathrm{~A}$ Annagür, ${ }^{1} \mathrm{~A}$ Hergüner, ${ }^{4} \mathrm{R}$ Örs. 'Department of Child and Adolescent Psychiatry; ${ }^{2}$ Department of Psychiatry, Konya University, Meram Medical Faculty; ${ }^{3}$ Department of Neonatology, Selcuk University, Selcuklu Medical Faculty; ${ }^{4}$ Department of Neonatology, Konya University, Meram Medical Faculty, Konya, Turkey

Objective There are contradictory findings in the literature about the effect of delivery type on postpartum depression and mother infant interaction.

Aim The aim of this study was to investigate the relationship between delivery mode, postpartum depression and maternal attachment.

Method Forty vaginal delivery (VD) women and 40 cesarean delivery (SD) women were recruited to participate in the study. The Edinburgh Postpartum Depression Scale (EPDS) was used to screen depressive symptoms, and Maternal Attachment Scale (MAS) was used to detect maternal attachment. Social support was assessed by the Multidimensional Scale of Perceived Social Support (MSPSS).

Results We found no significant difference in postpartum depression and perceived social support between two groups. On the other hand, MAS scores were significantly lower in SD women.

Discussion Delivery mode had no impact on the development of postpartum depression. However SD may be associated with impaired maternal attachment.

\section{POSTPARTUM DEPRESSION IN MOTHERS OF INFANTS WITH VERY LOW BIRTH WEIGHT}

doi:10.1136/archdischild-2012-302724.1290

'S Hergüner, ${ }^{2} \mathrm{~A}$ Annagür, ${ }^{3} \mathrm{E}$ Çiçek, ${ }^{4} \mathrm{H}$ Altunhan, ${ }^{5} \mathrm{R}$ Örs. 'Department of Child and Adolescent Psychiatry, Konya University, Meram Medical Faculty; ${ }^{2}$ Department of
Neonatology, Selcuk University, Selcuklu Medical Faculty; ${ }^{3}$ Department of Psychiatry, Konya University, Meram Medical Faculty; ${ }^{4}$ Department of Neonatology, Abant Izze Baysal University, Medical Faculty; ${ }^{5}$ Department of Neonatology, Konya University, Meram Medical Faculty, Konya, Turkey

Objective Giving birth to an infant with very low birth weight (VLBW) is a major life event for a mother. Several studies have shown that mothers of these infants are at greater risk of psychological distress. The aim of this study was to investigate the level of depressive symptoms and to determine the associated factors among mothers who have infants with VLBW.

Methods The sample consisted of 105 subjects: 35 mothers of VLBW infants $(<1500 \mathrm{~g}), 35$ mothers of low birth weight (LBW) infants (1500-2500 g), and 35 mothers of healthy term infants (>2500 g). The Edinburgh Postpartum Depression Scale (EPDS) was used to detect maternal depressive symptoms. Maternal social support was assessed by the Multidimensional Scale of Perceived Social Support (MSPSS).

Results The mean EPDS score and the number of mothers with high depressive scores (EPDS $>12$ ) were significantly higher in mothers of infants with VLBW than in mothers of LBW and term infants. EPDS score was negatively correlated with birth weight, gestational age, and perceived social support and positively correlated with duration of hospital stay in mothers of infants with VLBW. Low birth weight and long hospital stay were found as predictors of postpartum depression in mothers of infants with VLBW.

Conclusion The birth and subsequent hospitalization of an infant with very low birth weight evoke psychological distress in mothers. Pediatricians should be more careful about depressive symptoms of mothers of infants with VLBW and should refer for counseling when it is necessary.

\section{MATERNAL ATTACHMENT AND POSTPARTUM DEPRESSION IN MOTHERS OF PRETERM INFANTS AND THE ROLE OF ATTACHMENT STYLE}

doi:10.1136/archdischild-2012-302724.1291

'S Hergüner, ${ }^{2} \mathrm{~A}$ Annagür, ${ }^{3} \mathrm{E}$ Çiçek, ${ }^{1} \mathrm{~A}$ Hergüner, ${ }^{4} \mathrm{R}$ Örs. ${ }^{1}$ Department of Child and Adolescent Psychiatry, Konya University, Meram Medical Faculty; ${ }^{2}$ Department of Neonatology, Selcuk University, Selcuklu Medical Faculty; ${ }^{3}$ Department of Psychiatry; ${ }^{4}$ Department of Neonatology, Konya University, Meram Medical Faculty, Konya, Turkey

Background Preterm birth is a psychologically distressing experience for mothers of Preterm infants and a risk for the early mother - infant relationship.

Aims The aim of this study was to investigate the depressive symptoms and maternal attachment, and role of attachment style in mothers of preterm infants.

Subjects The study group consisted of 75 mothers of Preterm infants and the control group consisted of 75 mothers of full-term healthy infants. Outcome measures: Adult Attachment Scale (AAS) was used to determine the attachment style of the mother. Maternal depressive symptoms were screened by the Edinburgh Postpartum Depression Scale (EPDS), and maternal attachment was measured by the Maternal Attachment Inventory (MAI). Maternal social support was assessed by using the Multidimensional Scale of Perceived Social Support (MSPSS).

Results The mean EPDS score and the number of mothers with high depressive scores (EPDS > 12) were significantly higher and maternal attachment scores were lower in mothers of preterm infants than mothers of term infants. Mothers of preterm infants with insecure attachment style had higher EPDS and lower maternal attachment scores.

Conclusions Our findings suggest that postpartum depression and insecure attachment style of mothers may be a risk for maternal attachment in preterm infants' mothers. It would be important to screen depressive symptoms and detect attachment styles in 